\title{
Stone-age strategies and space-age media: Sex differences in sexual signaling on Facebook
}

\author{
IGOR MIKLOUŠIĆ1 \\ MIA KARABEGOVIĆ2 \\ LUKRECIJA PULJIĆ 3 \\ ${ }^{1}$ Institute of Social Sciences Ivo Pilar, Zagreb \\ ${ }^{2}$ Department of Cognitive Science, \\ Central European University, Budapest \\ ${ }^{3}$ Faculty of electrical engineering and computing, \\ University of Zagreb

\section{Correspondence:} \\ Igor Mikloušić \\ E-mail: igor.miklousic@pilar.hr
}

Keywords: Sexual signaling; evolutionary psychology; Facebook, sex differences; mate selection
Received October 30, 2017.

Revised December 12, 2017.

Accepted December 27, 2017.

\begin{abstract}
Background and purpose: Building on the proposition of the Sexual Strategies Theory that sex differences in mating strategies and intrasexual competition will be reflected in sexual signaling behavior towards possible mates, we sought to examine if such strategies would be observable on social networking sites.
\end{abstract}

Materials and methods: For the purpose of the study, ten male and ten female public profile pictures were randomly selected from a large pool of users $(N=1386)$ who chose to participate in the study and subscribed to a Facebook page created in order to aggregate users with an interest in Evolutionary psychology. Selected profile photos were then included in an online evaluation protocol, filled out by 31 independent raters, resulting in a total of 620 ratings. The protocol addressed nine evolutionarily relevant partner choice characteristics; (1) physical strength or athleticism, (2) access to resources or material possessions, (3) ambition or industriousness, (4) social status, (5) intelligence, (6) features of physical appearance, (7) features accentuating youthfulness, (8) high activity level, and (9) firtatious behavior.

Results: Males more frequently emphasized cues of social status, ambition and access to material resources, whereas females tended to emphasize features of physical appearance and of youthfulness. Furthermore, the perception of masculinity was mostly tied to the display of resources and physical strength, as was femininity to physical appearance and firtatious behavior.

Conclusions: The Sexual Strategies Theory predictions of mating display behaviors were confirmed in online settings, demonstrating the robustness of sex differences in mating-related behaviors.

\section{INTRODUCTION}

Grom the sudden rise in popularity of various social networking sites,

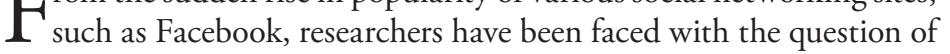
how psychological motives and mechanisms influence and reveal themselves in an online, virtual context. Research concerning Facebook has, so far, been concerned mostly with the construction of personalities of its users $(1,2)$ and user's self-presentation (3). However, little has been done to explain behavior on social networking sites in a broader evolutionary context. Given this lack of research, we sought to investigate the applicability of evolutionary hypotheses in explaining behavior in an online setting. More specifically, we wanted to examine whether the well-documented sex differences in sexual signaling in humans would be present in Facebook profile pictures. 
The shift towards using evolutionary biological theories in explaining and predicting human behavior is a characteristic of a somewhat new theoretical perspective within psychology, conveniently referred to as evolutionary psychology. It conceives of the human mind as an "adaptive toolbox" which was shaped by natural and sexual selection in order to efficiently solve adaptive problems faced by our ancestors (4). This view of human psychology builds thoroughly upon existing evolutionary biological theories (e.g. parental investment theory, as postulated by Trivers (5) and aims at rooting our understanding of psychology in evolutionary processes. This development was predicted by Darwin $(6, p .428)$ himself in the Origin of Species, when he prophetically stated that "Psychology will be based on a new foundation, that of the necessary acquirement of each mental power and capacity by gradation."

Notable contributions have been made so far in, among others: understanding our domestic violence (7), aggression (8); as well as some seemingly fundamentally human characteristics such as religiosity (9) and consumer behavior (10). The most significant progress, however, has undoubtedly been made in the domain of mating and mate choice. Large cross-cultural studies have shown universal and robust sex differences in mating strategies (11) and mate preferences $(12 ; 13)$ that conform to predictions stemming from an evolutionary framework. For instance, drawing predictions from the evolutionary concepts such as parental investment, parental uncertainty or reproductive capacity, Buss (12) was able to identify five evolutionary-backed predictions regarding mate preferences. The study showed that across the majority of 37 surveyed cultures, women tend to value resource-related factors such as financial capacity, ambition, and industriousness more than men, who on the other hand put more significance on reproduction-related traits such as physical attractiveness and youthfulness.

Only a limited number of studies has so far dealt with how these evolutionarily determined preferences and behaviors map onto behavior in online settings. Some prominent exceptions include Toma and Hancock's (14) study, which showed that it was possible to predict deceptive practices of users on online dating sites. As predicted by evolutionary theory, less attractive users tend to strategically increase their attractiveness by either manipulating their photographs or by lying about their appearance (height, weight) and age. Photographic self-enhancement was also more prevalent in females as, presumably, men tend to put more weight on physical attractiveness cues in mate selection. Furthermore, Tifferet and Vilnai-Yavetz (15) looked at sex differences in self-presentation on Facebook looking at both profile pictures, and cover photos, of 550 randomly selected Facebook profiles. Using wellestablished findings on the differential prevalence of certain behaviors between the sexes (e.g., risk-taking in men, emotional expressiveness in women), authors made detailed predictions about the expected content of users' Facebook profile and cover photos. Results confirmed their evolutionary psychology informed hypothesis that men accentuated their status by more frequently posing with either an object or wearing formal clothing. Men also displayed riskier behaviors, like participating in outdoor activities, presumably to signal adventurousness and sensation-seeking. Women, on the other hand, often demonstrated more positive and emotional facial cues, such as smiling and eye contact, which authors interpreted as an attempt to signal femininity. These differences were not found in Facebook cover photos, which the authors presume are used to show a wider social context.

Our study further explores these issues by using the sexual signaling framework. Building upon the sexual strategies theory (16) and the research on intrasexual competition and mate-attraction tactics (17), we expected women to be more likely to exhibit cues related to fertility, reproductive potential and youth; such as a healthy physical appearance, accentuating slim figures, fuller lips, clear skin, luscious hair, flirtatious behavior or high activity levels (H1). Men, on the other hand, were expected to exhibit signs of social status, access to resources and material possessions, intelligence, ambition, and athleticism (H2).

Facebook is a social networking site that presents selfgenerated content to a wide audience of other users and is thus subject to the same self-presentational motivations as all human behavior. However, it is a novel tool which utilizes technologies that have been unavailable for most part of human history. With this study, we wanted to explore the robustness of evolutionary psychological mechanisms in this novel environment.

\section{MATERIAL AND METHODS}

In order to avoid bias related to using only student Facebook profiles (e.g. 3), we devised a new method that utilized social networking sites, specifically Facebook for obtaining a diverse sample. A Facebook page (www.facebook.com/EvolucijskaPsihologija/) was created in order to aggregate users with an interest in Evolutionary psychology. They were mostly gathered through requests and suggestions by other members of the community. Subsequently, participants were recruited through a virtual Facebook "event" that served the purpose of informing the group of the purpose of the study. After choosing the option to "attend" the event, a private message was sent to each participant with a detailed description of the research aims in order to obtain their consent. A sample of ten male and ten female profile pictures was randomly selected from a large pool of users $(\mathrm{N}=1386)$ who chose to participate in the study, and downloaded to the researcher's hard drive with coded file names, without any additional information which could serve to identify the users in order to ensure anonymity.

The profile photos were then included in an online evaluation protocol, using the Survey Gizmo online pooling platform, that was filled out by independent raters. 
Table 1. Discriminative variables and the discriminative function correlation structure matrix $(N=20)$.

\begin{tabular}{|lc|}
\hline Variable & Function \\
\hline Features accentuating youthfulness & -.495 \\
Features of physical appearance & -.470 \\
Ambition or industriousness & .455 \\
Access to resources or material possessions & .455 \\
Social status & .399 \\
Flirtatious behavior & -.276 \\
Physical strength or athleticism & .223 \\
High activity level & .173 \\
Intelligence & .088 \\
\hline
\end{tabular}

The raters were 31 volunteers, recruited through personal contact ( 16 female, 15 male, $\mathrm{M}$ age $=33$ ) amounting to a total of 620 profile photo ratings.

A rating protocol addressing evolutionarily relevant partner choice characteristics was adapted according to Buss and Schmitt (16) for the purposes of the study. Raters were first shown a brief explanation of the nine criteria and given a few examples of how these could be accentuated visually. They were then asked to rate each of the twenty profile pictures, presented in a randomized order, on how much they focused on the specific traits. The ratings were done on Likert scales ranging from 1 (the characteristic is not accentuated at all) to 4 (the characteristic is accentuated to a high degree). The rating criteria used were: (1) physical strength or athleticism, (2) access to resources or material possessions, (3) ambition or industriousness, (4) social status, (5) intelligence, (6) features of physical appearance, (7) features accentuating youthfulness, (8) high activity level, and (9) flirtatious behavior.

A measure of inter-rater agreement (ICC) was calculated for the nine evolutionary-relevant traits, for each photograph. The values of the average agreement measures ranged from .425 to .890 , with the average of 0.737 .

\section{RESULTS}

We conducted the analysis on the mean evaluations of 31 raters for every category on the 20 selected profiles. Discriminant analysis was used in order to examine if any of the proposed categories can be used to discern between male and female profiles. Thus, the sex of the Facebook profile picture owner was the grouping variable while the discriminant variables were ratings on nine evolutionary relevant variables included in the survey; (1) physical strength or athleticism, (2) access to resources or material possessions, (3) ambition or industriousness, (4) social status, (5) intelligence, (6) features of physical appearance, (7) features accentuating youthfulness, (8) high activity level, and (9) flirtatious behavior.

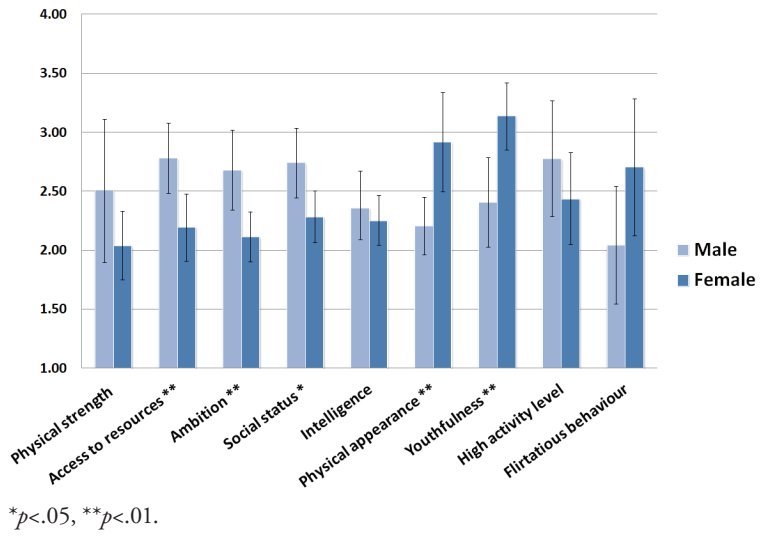

Figure 1. Mean evaluations of nine evolutionarily-relevant variables across sex (error bars represent standard deviations).

The assumption of a multivariate normal distribution was examined using the Box's $M$ tests, that proved nonsignificant $(M=138.678, F=1.342$; $d f 1=45 ; d f 2=1064.399$; $p=.067$ ), demonstrating homogeneity of the covariance matrices which is the prerequisite for the use of discriminant analysis. The extracted canonical function (Eigenvalue $=2.771$ ) explained $73.44 \%$ of the "sex" variance, with a canonical correlation of .857. Wilks' $\lambda=.265$ was statistically significant $\left(\chi^{2}=17.919, d f=9 ; p=.036\right)$.

Table 1 shows the correlation structure matrix which describes the saturation of each of the examined variables with the discriminative function. As correlations of .30 or above commonly considered relevant we can single out the following discriminant variables: Features accentuating youthfulness $(\mathrm{r}=-.495)$, Features of physical appearance $(\mathrm{r}=-.470)$, Ambition or industriousness $(\mathrm{r}=.455)$, Access to resources or material possessions ( $\mathrm{r}=.455)$, and Social status $(\mathrm{r}=.399)$. The group centroid value for the profile picture belonging to the female sex was -1.579 and 1.579 for the male sex. Table 1 thus demonstrates that features accentuating youthfulness and features accentuating physical appearance were related to female profile pictures; whereas ambition or industriousness, access to resources or material possessions and social status to male ones.

Sex differences in the emphases on different traits are visually presented in Figure 1: notably, there is an almost non-existent difference in intelligence signaling between the sexes.

Finally, in order to see to what extent the chosen variables can be used to discern the sex of Facebook users, we calculated the efficiency of sex classification using these traits. The classification of female profiles was somewhat more efficient $(80 \%)$ than the classification of male profiles $(70 \%)$.

\section{DISCUSSION}

The primary focus of this study was to test evolutionary-psychology-based predictions about self-presentation via profile photos on Facebook. We sought to investigate 
how men and women make use of this self-presentational tool to accentuate different traits: those that are most highly valued by the opposite sex as they mirror different adaptive problems they faced in our evolutionary past.

Our results provide support for most of these predictions. Specifically, we show that men were more likely to accentuate access to resources, social status, ambition and industriousness, as displayed by photographs taken in foreign countries (as a possible cue of access to resources) or in formal attire in business or elite settings (as a cue of social status, ambition as well as the aforementioned access to resources). Female Facebook users more often accentuated youthful features and physical appearance. In most cases, this included close-up photographs of faces, sometimes digitally manipulated to enhance the appearance of clear skin, full lips, and the like. This tendency (digital enhancement) of females to "touch up" the photographs used in visual self-presentation has also been found in studies of photographs uploaded to online dating sites (14).

The differences in signaling between the sexes proved to be robust, as shown in the success of classifying profile photos as male and female using the included variables. Such big effects are not unusual in psychological studies using evolutionary theory, as the underlying mechanisms are largely considered to be universal and "hard-wired". However, we need to point out that not all the predictions were borne out. Intelligence was one of the traits accentuated equally by men and women. Even though earlier work has classified this trait as primarily desirable for men (16), there have been claims that it is just as important for both sexes (18), but in different contexts. For instance, while short-term mating strategies show a difference in this preference, with men being less concerned with their potential partners' intelligence, it is considered equally desirable by both men and women when it comes to longterm mating strategies (19).

Two further traits that did not reach the expected level of significance were physical ability (for males) and high activity level (for females). These have the common denominator of having, one way or another, to do with movement, and it is unclear how they could be differentiated using a single photograph. Confusion as to which constitutes which could have resulted in an overlap between these dimensions, leading to lowering their value as predictors of the respective profile owners' photographs. Finally, flirtatious behavior was only marginally significant $(p=.067)$. It is not excluded that it would have reached the conventional levels of significance given a larger sample size, but it also suffers from a similar problem as the aforementioned physical ability and high activity, in that it is more difficult to relate using only a profile picture as it includes, to no small degree, verbal communication and body language which do not lend themselves to easy transfer into a static visual medium.

Finally, there are several variables beyond the scope of this study whose relationship to self-presentation would be relevant to explore from an evolutionary perspective. Pro- file owners' relationship status and preference for short- or long-term mating strategies is one avenue which, form an evolutionary psychology perspective, might factor into which traits are being featured most prominently. Furthermore, the type and scope of the audience one is communicating with through one's Facebook account might also influence certain aspects of self-presentational behavior (for instance, if one uses it exclusively to keep in touch with family members, the input relevant for activating intrasexual competition mechanisms might be absent). Having now established that evolutionary mechanisms play a role in self-presentation on online social networks, the constraints and subtle nuances of the behavior in question should be looked at with a more fine-grained approach in order to elucidate just how big a proportion of a seemingly novel set of behaviors can be explained by using parameters informed by our "stone age minds" (4).

\section{REFERENCES}

1. WILSON K, FORMASIER S, WHITE K M 2010 Psychological predictors of young adults' use of social networking sites. Cyberpsychol Behav 13: 173-177. https://doi.org/10.1089/cyber.2009.0094

2. RYAN T, XENOS S 2011 Who uses Facebook? An investigation into the relationship between the Big Five, shyness, narcissism, loneliness, and Facebook usage. Comput Hum Beh 27: 1658-1664. https://doi.org/10.1016/j.chb.2011.02.004

3. BACK M D, STOPFER J M, VAZIRE S, GADDIS S, SCHMUKLE S C, EGLOFF B, GOSLING S D 2010 Facebook profiles reflect actual personality, not self-idealization. Psychol Sci 21: 372-374.

4. COSMIDES L, TOOBY J 1997 Evolutionary psychology: A primer.

5. TRIVERS R, 1972 Parental investment and sexual selection 136: Biological Laboratories, Harvard University. Cambridge, MA, p 179

6. DARWIN C 1859 On the Origin of Species by Means of Natural Selection. John Murray, London

7. DALY M, WILSON M 1988 Homicide, Transaction Publishers, Vancouver

8. BUSS D M, SHACKELFORD T K 1997 From vigilance to violence: mate retention tactics in married couples. J Pers Soc Psychol 72, 346-361. https://doi.org/10.1037/0022-3514.72.2.346

9. SOSIS R, ALCORTA C 2003 Signaling, solidarity, and the sacred: The evolution of religious behavior. Evol Anthropol 12: 264-274. https://doi.org/10.1002/evan.10120

10. SAAD G, GILL T, 2000 Applications of evolutionary psychology in marketing. Psychol Market 17.1005-1034.

11. SCHMITT D P 2003 Universal sex differences in the desire for sexual variety: tests from 52 nations, 6 continents, and 13 islands. J Pers Soc Psychol 85: 85-104.

https://doi.org/10.1037/0022-3514.85.1.85

12. BUSS D M 1989 Sex differences in human mate preferences: Evolutionary hypotheses tested in 37 cultures. Behav Brain Sci 12, 1-14. https://doi.org/10.1017/S0140525X00023992

13. SHACKELFORD T K, SCHMITT D P, BUSS D.M 2005 Universal dimensions of human mate preferences. Pers Ind Differ 39: 447-458.

14. TOMA C L, HANCOCK J T 2010 Looks and lies: The role of physical attractiveness in online dating self-presentation and deception. Commun Res 37: 335-351. https://doi.org/10.1177/0093650209356437 
15. TIFFERET S, VILNAI-YAVETZ I 2014 Gender differences in Facebook self-presentation: An international randomized study. Comput Hum Behav 35: 388-399. https://doi.org/10.1016/j.chb.2014.03.016

16. BUSS D M, SCHMITT D P 1993 Sexual Strategies: An Evolutionary Perspective on Human Mating. Psychol Rev 100: 204-232. https://doi.org/10.1037/0033-295X.100.2.204

17. BUSS D M 1988 The Evolution of Human Intrasexual Competition: Tactics of Mate Attraction. J Pers Soc Psychol 54, 616-628. https://doi.org/10.1037/0022-3514.54.4.616
18. MILLER G 1999 Sexual selection for indicators of intelligence. In Novartis Foundation Symposium. John Wiley, Chichester, New York, p 260-270

19. KENRICK D T, SADALLA E K, GROTH G, TROST M R 1990 Evolution, traits, and the stages of human courtship: Qualifying the parental investment model. J Pers, 58, 97-116.

20. BURNS R., BURNS R. (2008), Business Research Methods and Statistics using SPSS, chapter 25, Discriminant Analysis, SAGE Publications Ltd., California, USA 